\title{
Downregulation of lipolysis-stimulated lipoprotein receptor promotes cell invasion via claudin-1-mediated matrix metalloproteinases in human endometrial cancer
}

\author{
HIROSHI SHIMADA $^{1,2}$, SEIRO SATOHISA ${ }^{1}$, TAKAYUKI KOHNO ${ }^{2}$, TAKUMI KONNO ${ }^{2}$, \\ KEN-ICHI TAKANO ${ }^{3}$, SYUNTA TAKAHASHI ${ }^{2}$, TSUBASA HATAKEYAMA ${ }^{2}$, \\ CHIHIRO ARIMOTO ${ }^{2}$, TSUYOSHI SAITO $^{1}$ and TAKASHI KOJIMA ${ }^{2}$
}

\begin{abstract}
Departments of ${ }^{1}$ Obstetrics and Gynecology and ${ }^{2}$ Cell Science, Research Institute for Frontier Medicine;
${ }^{3}$ Department of Otolaryngology, Sapporo Medical University School of Medicine, Sapporo, Hokkaido 060-8556, Japan
\end{abstract}

Received February 9, 2016; Accepted July 5, 2017

DOI: $10.3892 / \mathrm{ol} .2017 .7038$

\begin{abstract}
Lipolysis-stimulated lipoprotein receptor (LSR) is a novel molecule present at tricellular contacts which recruits tricellulin (TRIC), a molecular component of tricellular tight junctions (tTJs). LSR and TRIC are colocalized with the bicellular tight junction (bTJ) protein claudin (CLDN)-1-based tight junction strands at tricellular corners. Knockdown of LSR in normal epithelial cells affects tTJ formation and the epithelial barrier function. In cancer cells knockdown of LSR has been demonstrated to increase cell invasion. However, the detailed mechanisms of how the downregulation of LSR enhances cell invasion in cancer remain unclear. In the present study, knockdown of LSR by small interfering RNA (siRNA) in Sawano human endometrial adenocarcinoma cells induced cell invasion. In LSR-knockdown Sawano cells, upregulation of CLDN-1 protein, which contributes to the cell invasion via matrix metalloproteinases (MMPs), was observed compared with the control group by western blotting and immunostaining. Knockdown of LSR significantly induced Sp1 transcription factor activity in the CLDN-1 promoter region. In LSR-knockdown Sawano cells, DNA microarray analysis demonstrated that MMP-1, MMP-2 and MMP-10 mRNA levels were increased, and the protein levels of membrane-type 1-MMP, MMP-2, MMP-9 and MMP-10 were shown to be increased on western blots. Knockdown of CLDN-1 with siRNA prevented the upregulation of cell invasion induced by the knockdown of LSR in Sawano cells. On the invasive front
\end{abstract}

Correspondence to: Dr Takashi Kojima, Department of Cell Science, Research Institute for Frontier Medicine, Sapporo Medical University School of Medicine, South-1, West-17 Chuo-ku, Sapporo, Hokkaido 060-8556, Japan

E-mail:ktakashi@sapmed.ac.jp

Key words: endometrial cancer, cell invasion, lipolysis-stimulated lipoprotein receptor, claudin-1, matrix metalloproteinases, Sp1 transcription factor of human endometrial carcinoma tissue samples, a decrease in LSR and increase in CLDN-1 protein levels were observed using immunohistochemical methods. In conclusion, the results indicate that the downregulation of LSR promotes cell invasion of human endometrial cancer via CLDN-1 mediation of MMPs. This mechanism is important for studying the association of tTJs with the cellular invasion of cancer.

\section{Introduction}

Tricellular tight junctions (tTJs) form at the convergence of bicellular tight junctions (bTJs) where three epithelial cells meet in polarized epithelia (1). Lipolysis-stimulated lipoprotein receptor (LSR) was identified as a novel molecular component of tricellular contacts that is localized on the majority of epithelial tissues (2). LSR is required for normal tTJ formation and provides a high barrier function for the cellular sheet. LSR recruits tricellulin (TRIC), which is a molecular component of tTJs (1). A previous study showed that LSR and TRIC are colocalized with the bTJ protein claudin (CLDN)-based tight junction (TJ) strands reconstituted in CLDN-1-overexpressing mouse L fibroblasts (2).

TJs are involved in signal transduction mechanisms that regulate epithelial cell proliferation, gene expression, differentiation and morphogenesis (3). Loss of TJs compromises cellular polarity and stimulates dedifferentiation $(4,5)$. Furthermore, loss of several TJ proteins enhances tumor progression (6), and downregulation of CLDN-7 promotes cell invasion in endometrial cancer (7).

The overexpression of certain TJ proteins, including CLDNs, is associated with tumor growth and metastasis (8). In addition, the overexpression of CLDN-1 enhances cell invasion via the activation of matrix metalloproteinases (MMPs) in certain cancer types $(9,10)$. TRIC expression is reduced in hepatic fibrolamellar carcinoma and tonsillar squamous cell carcinoma as compared with normal tissues $(11,12)$, and also demonstrates a significant negative correlation with the degree of differentiation in pancreatic cancer (13). Furthermore, TRIC expression in gastric carcinoma cells is negatively regulated by Snail-induced epithelial-mesenchymal transition (EMT) (14). 
Knockdown of LSR has been demonstrated to increase cell motility and invasion in bladder cancer cells (15). LSR signaling also promotes aggressive/tumor-initiating cell behaviors in breast cancer (16). In addition, our recent study revealed that small interfering RNA (siRNA)-mediated knockdown of LSR promoted cell invasion in human endometrial cancer cells. Although LSR expression level is associated with tumor progression (15), it remains unclear how the knockdown of LSR enhances cancer cell invasion.

In the present study, knockdown of LSR with siRNA in human endometrial cancer Sawano cells induced cell invasion. In LSR-knockdown Sawano cells, upregulation of CLDN-1 and MMPs, as well as increased Sp1 transcription factor activity in the CLDN-1 promoter region, were observed. Knockdown of CLDN-1 with siRNA prevented the induction of cell invasion by the downregulation of LSR in Sawano cells. The aim of this study was to analyze the function of LSR in cell invasion via CLDN-1-mediated MMPs in human endometrial cancer.

\section{Materials and methods}

Antibodies. Rabbit polyclonal antibodies against TRIC (cat. no. 48-8400), occludin (OCLN; cat. no. 71-1500) and CLDNs-1 (cat. no. 71-7800), -3 (cat. no. 34-1700), -4 (cat. no. 36-4800) and -7 (cat. no. 34-9100) were obtained from Zymed Laboratories (Thermo Fisher Scientific, Inc., Waltham, MA, USA). A rabbit polyclonal anti-LSR antibody was obtained from Novus Biological USA (Littleton, CO, USA; cat. no. NBP1-89631). A mouse monoclonal anti-LSR antibody was obtained from Abnova (Heidelberg, Germany; cat. no. H00051599-K). Rabbit monoclonal antibodies against membrane-type 1 (MT1)-MMP (cat. no. 13130), MMP-2 (cat. no. 4022) and MMP-9 (cat. no. 13667) were obtained from Cell Signaling Technology, Inc. (Tokyo, Japan). A rabbit polyclonal anti-MMP-10 antibody was obtained from Abcam (Cambridge, UK; cat. no. ab199688). A rabbit polyclonal anti-actin antibody was obtained from Sigma-Aldrich (Merck Millipore, St. Louis, MO, USA; cat. no. A2066). Alexa Fluor 488 (green)-conjugated anti-rabbit IgG and Alexa Fluor 594 (red)-conjugated anti-mouse IgG antibodies were purchased from Molecular Probes, Inc. (Thermo Fisher Scientific, Inc.). Horseradish peroxidase-conjugated anti-mouse and anti-rabbit IgG antibodies were from Cell Signaling Technology, Inc. (cat. nos. 7074 and 7076).

Cell line culture and treatment. The human endometrial cancer cell line Sawano (RCB1152) was purchased from RIKEN BioResource Center (Tsukuba, Japan). Sawano cells were maintained with minimal essential medium (MEM; Sigma-Aldrich; Merck Millipore) supplemented with $10 \%$ dialyzed fetal bovine serum (FBS; Thermo Fisher Scientific, Inc.). The medium contained $100 \mathrm{U} / \mathrm{ml}$ penicillin, $100 \mu \mathrm{g} / \mathrm{ml}$ streptomycin and $50 \mu \mathrm{g} / \mathrm{ml}$ amphotericin-B. Sawano cells were plated on 35 and $60-\mathrm{mm}$ culture dishes, which were coated with rat tail collagen $(500 \mu \mathrm{g}$ dried tendon $/ \mathrm{ml}$ in $0.1 \%$ acetic acid) and incubated in a humidified incubator with $5 \% \mathrm{CO}_{2}$ at $37^{\circ} \mathrm{C}$. The Sawano cells were transfected with siRNAs against LSR and CLDN-1 for $48 \mathrm{~h}$ prior to further observations being made. A scrambled siRNA sequence (BLOCK-iT Alexa Fluor fluorescent; Invitrogen; Thermo Fisher Scientific, Inc.) was used as control siRNA.
RNA interference and transfection. siRNA duplex oligonucleotides against LSR and CLDN-1 were synthesized by Thermo Fisher Scientific, Inc. The following siRNAs against LSR were used: siRNA-A forward, 5'-CCCACGCAACCCAUCG UCAUCUGGA-3'; siRNA-A reverse, 5'-UCCAGAUGACGA UGGGUUGCGUGGG-3'; siRNA-B forward, 5'-GGCCGG AGGAUUACCAUCAUCACCGGA-3'; siRNA-B reverse, 5'-UUCCGGUGAUGGUAAUCCUCCGGCC-3'; siRNA-C forward, 5'-CACGGACAGCAGUGCGGCCUCUGAA-3'; and siRNA-C reverse 5'-UUCAGAGGCCACACUGCUGUCCGU G-3'. The siRNA sequences against CLDN-1 were as follows: Forward, 5'-GACUCCUUGCUGAAUCUG-3'; and reverse, 5'-UCAGAUUCAGCAAGGAGU-3'.

At $24 \mathrm{~h}$ after plating, the Sawano cells were transfected with the three pairs of LSR siRNAs and the pair of CLDN-1 siRNAs (100 nM of each) using Lipofectamine ${ }^{\mathrm{TM}}$ RNAiMAX Reagent (Invitrogen; Thermo Fisher Scientific, Inc.).

Immunostaining. The cultured cells in Iwaki 35-mm glass coated wells (Asahi Techno Glass, Chiba, Japan) were fixed with cold acetone and ethanol $(1: 1)$ at $-20^{\circ} \mathrm{C}$ for $10 \mathrm{~min}$. Following rinsing with PBS, the cells were incubated with mouse monoclonal anti-LSR (1:100) and rabbit polyclonal anti-CLDN-1 (1:100) antibodies at room temperature for 1 h. Alexa Fluor 488-conjugated anti-rabbit IgG and Alexa Fluor 594-conjugated anti-mouse IgG (1:200) were used as secondary antibodies. The specimens were examined using an epifluorescence microscope (Olympus Corporation, Tokyo, Japan) and a confocal laser scanning microscope (LSM5; Carl Zeiss, Jena, Germany).

Western blot analysis. The cultured cells were scraped from a 60 -mm dish containing 400 or $600 \mu \mathrm{l}$ buffer $\left(1 \mathrm{mM} \mathrm{NaHCO}_{3}\right.$ and $2 \mathrm{mM}$ phenylmethylsulfonyl fluoride), transferred to microcentrifuge tubes, and then sonicated for $10 \mathrm{sec}$. The protein concentrations of the samples were determined using a Pierce BCA Protein Assay kit (Thermo Fisher Scientific, Inc.). Aliquots of $15 \mu \mathrm{l}$ of protein for each sample were separated by electrophoresis on 5-20\% SDS polyacrylamide gels (Wako Pure Chemical Industries, Ltd., Osaka, Japan), and electrophoretic transfer to a nitrocellulose membrane (Immobilon; Merck Millipore) was performed. The membrane was saturated for $>30 \mathrm{~min}$ at room temperature with blocking buffer $(25 \mathrm{mM}$ Tris, $\mathrm{pH} 8.0,125 \mathrm{mM} \mathrm{NaCl}, 0.1 \%$ Tween-20, and 4\% skimmed milk) and incubated with polyclonal rabbit anti-LSR $(1: 1,000)$, anti-TRIC (1:1,000), anti-OCLN (1:1,000), anti-CLDN-1, -3, -4 and $-7(1: 1,000)$ and anti-actin $(1: 1,000)$ antibodies at room temperature for $>1 \mathrm{~h}$. Then the membrane was incubated with horseradish peroxidase-conjugated anti-mouse and anti-rabbit IgG antibodies $(1: 2,000)$ at room temperature for $1 \mathrm{~h}$. The immunoreactive bands were detected using an ECL western blotting system (RPN2232; GE Healthcare, Chicago, IL, USA). The quantification of the bands was performed using Image J software 1.48 (National Institutes of Health, Bethesda, MD, USA).

Matrigel invasion assay. For the invasion assay, Matrigel (BD Biosciences, Bedford, MA, USA) and Cell Culture Inserts (pore size $8 \mu \mathrm{m}$; BD Biosciences) were used. Sawano cells were plated using MEM without FBS onto the upper Matrigel-coated 
chamber (354480; Corning Incorporated, Corning, NY, USA), and the lower chamber of the Transwell was filled with human fibroblast-conditioned medium containing $10 \mathrm{nM}$ epidermal growth factor as the chemoattractant. The cells were then incubated for $36 \mathrm{~h}$. Subsequent to this, the medium was removed, and the Matrigel-coated upper chamber was fixed with $100 \%$ methanol for $10 \mathrm{~min}$ and stained with Giemsa for $20 \mathrm{~min}$. The areas of invading cells were measured in $1,00,0000 \mu \mathrm{m}^{2}$ area by automatic binarization using a microscope imaging system (Olympus Corporation).

Dual-luciferase reporter assay. Sawano cells were placed in 96-well plates and cultured overnight. An Sp1 luciferase reporter gene construct or negative control (CCS-6027L; SABiosciences; Qiagen Inc., Germantown, MD, USA) and siRNA specific for LSR were cotransfected into the cells for $48 \mathrm{~h}$. Whole protein extracts were prepared and luciferase activity was measured using a Dual-Luciferase Assay Kit (Promega Corporation, Madison, WI, USA) according to the manufacturer's protocol.

GeneChip analysis. Microarray analyses were performed using a 3D-Gene Human Oligo chip 25k (Toray, Tokyo, Japan) and microarray images were automatically analyzed using $\mathrm{AROS}^{\mathrm{TM}}$, version 4.0 (Operon Biotechnologies, Tokyo, Japan).

Immunohistochemical analysis. This study was approved by the ethics committee of the Sapporo Medical University School of Medicine (Sapporo, Japan). Human endometrial carcinoma tissues were obtained from 15 patients with endometrial adenocarcinoma who underwent hysterectomy between January 2012 and December 2013 at Sapporo Medical University Hospital. Hematoxylin and eosin-stained slides from each case were reviewed, and the diagnosis and grades of the tumors were determined according to the guidelines of the World Health Organization classification (17). Gynecologists and pathologists at the hospital established the diagnosis of endometrial adenocarcinoma. All endometrial adenocarcinoma were classic endometrial type I.

Human endometrial cancer tissues were embedded in paraffin following fixation with $10 \%$ formalin in PBS. Briefly, $5-\mu \mathrm{m}$-thick sections were dewaxed in xylene, rehydrated in ethanol, and heated with Vision BioSystems Bond Max using ER2 solution (Leica Biosystems Nussloch GmBH, Nussloch, Germany) in an autoclave for antigen retrieval. Endogenous peroxidase was blocked by incubation with $3 \%$ hydrogen peroxide in methanol for $10 \mathrm{~min}$. The tissue sections were then washed twice with Tris-buffered saline (TBS) and preblocked with Block Ace (DS Pharma Biomedical Co., Ltd., Suita, Japan) for $1 \mathrm{~h}$. Subsequent to washing with TBS, the sections were incubated with rabbit polyclonal anti-LSR (1:100) and rabbit polyclonal anti-CLDN-1 (1:100) antibodies for $1 \mathrm{~h}$. The sections were then washed three times in TBS and incubated with Vision BioSystems Bond Polymer Refine Detection kit (DS9800; Leica Biosystems Nussloch GmBH). Following three washes in TBS, a diaminobenzidine tetrahydrochloride solution was applied. Finally, the sections were counterstained with hematoxylin. Immunoreactivity for LSR and CLDN-1 on the invasive front of human endometrial carcinoma was assessed.
Table I. List of probes for genes that were upregulated in Sawano cells transfected with small interfering RNA against lipolysis-stimulated lipoprotein receptor.

\begin{tabular}{lccc}
\hline Gene name & ID & GenBank ID & Fold-change \\
\hline MMP1 & H200007011 & NM_002421 & 1.90 \\
MMP2 & H200011747 & NM_004530 & 1.72 \\
MMP10 & H200000576 & NM_002425 & 2.14 \\
\hline
\end{tabular}

MMP, matrix metalloproteinase.

Statistical analysis. Each set of results represents at least three separate experiments. Results are presented as the mean \pm standard error of the mean. Differences between groups were tested by ANOVA followed by a post hoc test. $\mathrm{P}<0.01$ was considered to indicate a statistically significant difference.

\section{Results}

Knockdown of LSR enhances cellular invasion in Sawano cells. To investigate whether LSR expression contributed to the cellular invasion of endometrial cancer, knockdown of LSR was performed using siRNA in Sawano cells. In Sawano cells transfected with one of the three sets of siRNA against LSR, cellular invasion measured using the Matrigel invasion assay appeared to be enhanced compared to the control (Fig. 1A).

Knockdown of LSR increases CLDN-1 protein in Sawano cells. Alterations in the levels of other TJ proteins subsequent to knockdown of LSR in Sawano cells were investigated. As revealed by western blot analysis, $48 \mathrm{~h}$ after transfection with three sets of siRNAs (A, B and C) against LSR, LSR protein abundance was decreased and CLDN-1 protein significantly increased compared with the control $(\mathrm{P}<0.01)$, whereas no changes in TRIC, OCDN, CLDN-3, CLDN-4 or -7 abundance were observed (Fig. 1B). The increase in CLDN-1 protein level enhanced by knockdown of LSR was inversely associated with the value of LSR expression (Fig. 1B). Using immunostaining $48 \mathrm{~h}$ after transfection with the siRNA-C against LSR, LSR protein could no longer be detected at the subapical membranes and CLDN-1 protein abundance was increased at the subapical and basolateral membranes of cells (Fig. 1C).

Knockdown of LSR induces Spl activity in Sawano cells. The CLDN-1 promoter region contains an Sp1 binding site, and a mutation in the region results in a loss of CLDN-1 transcription (18). Since knockdown of LSR enhanced CLDN-1 protein in Sawano cells, the present study investigated the change in Sp1 activity in Sawano cells transfected with siRNA against LSR. Knockdown of LSR by siRNA-C significantly induced Sp1 activity in the Sawano cells (Fig. 1D).

Knockdown of LSR increases the expression of MMPs in Sawano cells. Overexpression of CLDN-1 enhances cell invasion of certain types of cancer via activation of MMPs, including MT1-MMP and MMP-2 $(9,10)$. To investigate 
A

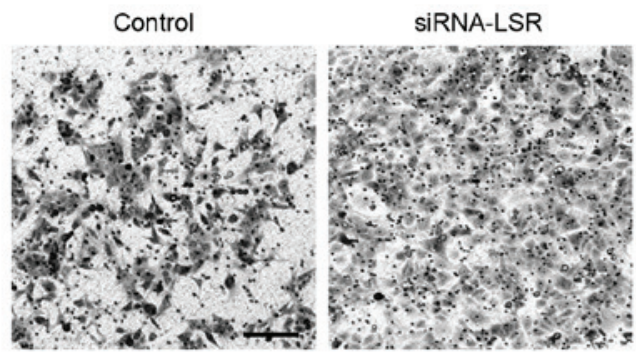

B

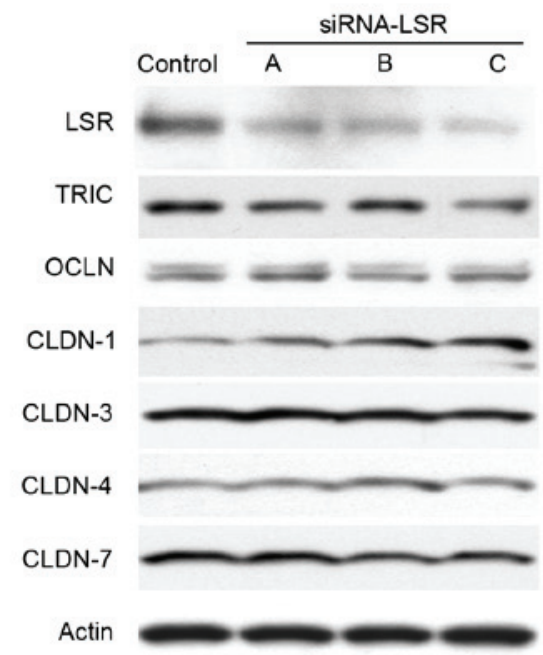

C
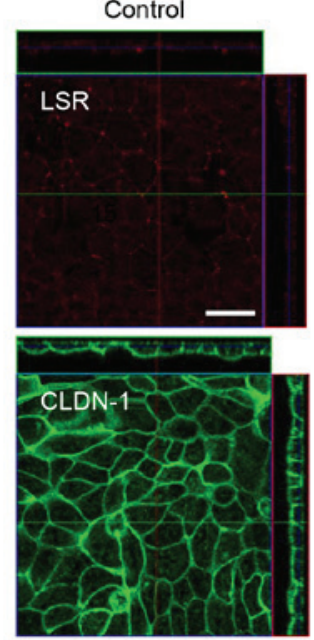

siRNA-LSR

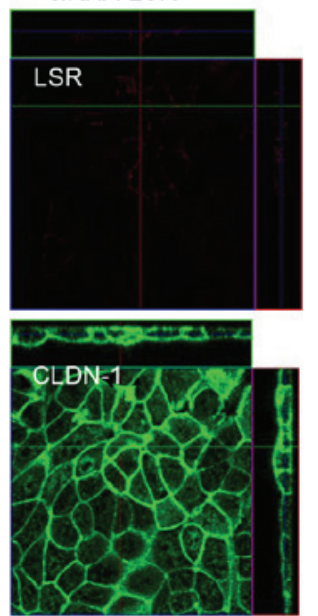

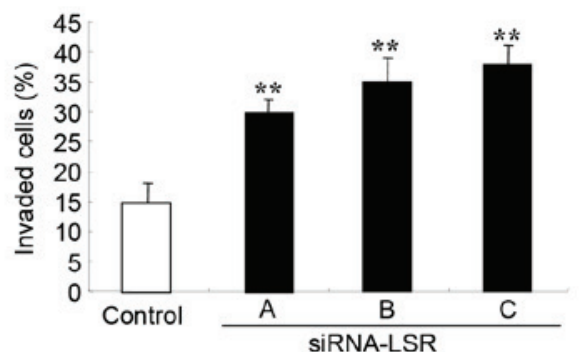

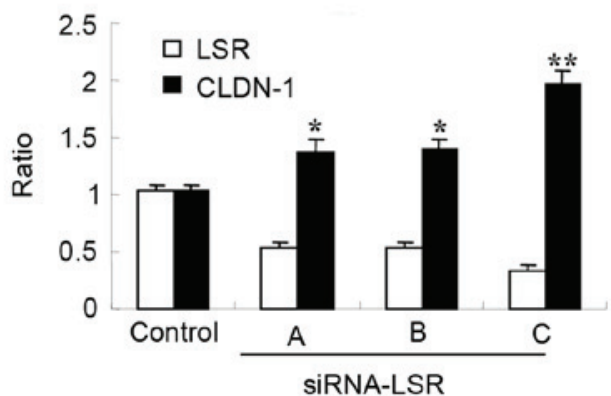

D

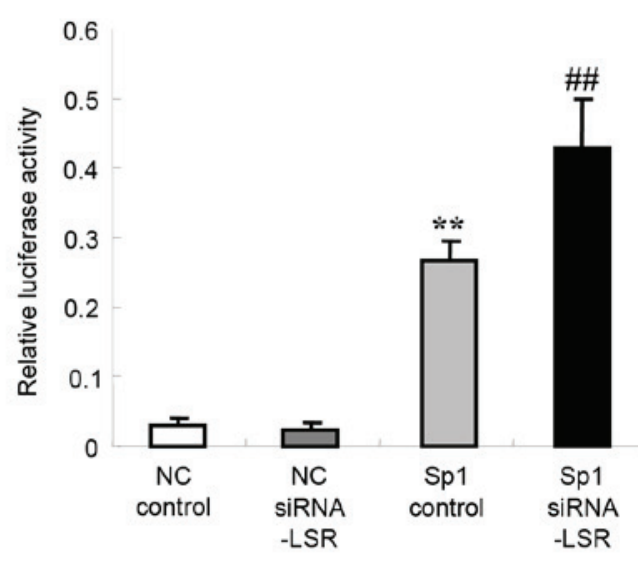

Figure 1. (A) Matrigel invasion assay of Sawano cells transfected with three sets of siRNA, A, B and C, LSR. Scale bar, $100 \mu \mathrm{m}$. The results are presented below as a histogram. Control vs. siRNA, ${ }^{* *} \mathrm{P}<0.01$. (B) Western blotting for LSR, TRIC, OCLN, CLDN-1, $-3,-4$ and -7 in Sawano cells transfected with three sets of siRNA against LSR. The results for the expression of LSR and CLDN-1 are presented as a histogram. Control vs. siRNA, ${ }^{*} \mathrm{P}<0.05,{ }^{* *} \mathrm{P}<0.01$. (C) Immunostaining of LSR and CLDN-1 in Sawano cells transfected with siRNA-C against LSR. Scale bar, $20 \mu \mathrm{m}$. (D) Sp1 transcription factor activity in

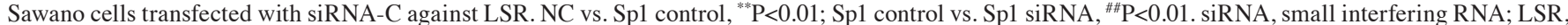
lipolysis-stimulated lipoprotein receptor; TRIC, tricellulin; OCLN, occludin; CLDN, claudin; NC, negative control.

whether upregulation of CLDN-1 by knockdown of LSR affected the expression of MMPs, DNA microarray analysis of Sawano cells transfected with siRNA against LSR was performed. In the DNA microarray, the mRNAs of MMP1, MMP2 and MMP10 were increased compared with the control (Table I). Next, using western blotting, changes to MT1-MMP, MMP-2, MMP-9 and MMP-10 levels following the knockdown of LSR were confirmed. The levels of MT1-MMP, MMP-2, MMP-9 and MMP-10 proteins in Sawano cells transfected with siRNA against LSR were increased compared with the control (Fig. 2A).

Knockdown of CLDN-1 prevents cell invasion induced by knockdown of LSR in Sawano cells. To investigate whether downregulation of CLDN-1 affected the upregulation of cell invasion induced by knockdown of LSR in Sawano cells, the cells were transfected with siRNAs against LSR and CLDN-1. In these cells, upregulation of CLDN-1 protein by knockdown 

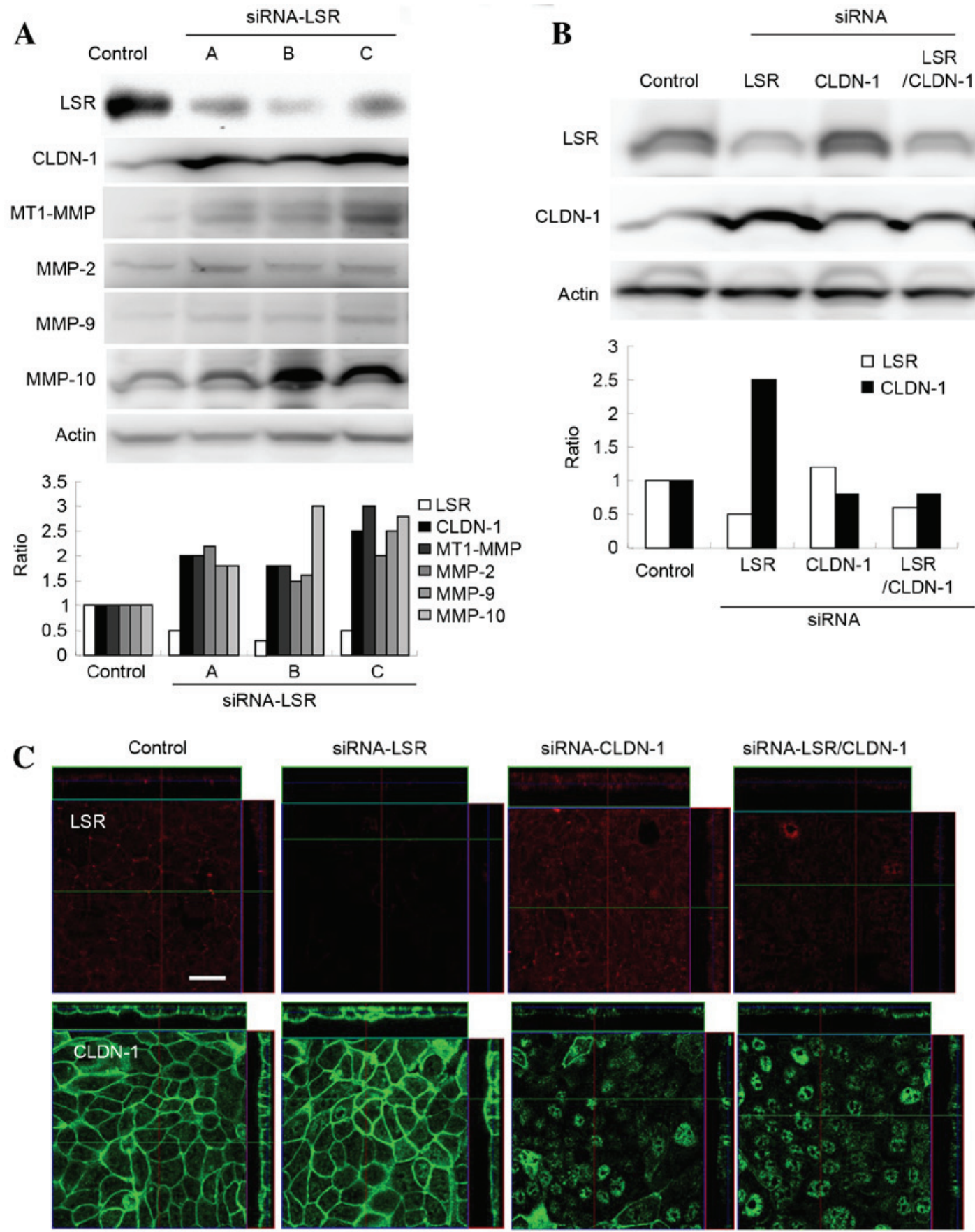

Figure 2. (A) Western blotting for LSR, CLDN-1, MT1-MMP, MMP-2, MMP-9 and MMP-10 in Sawano cells transfected with three sets of siRNA against LSR. The results are presented below as a histogram. (B) Western blotting for LSR and CLDN-1 in Sawano cells transfected with or without siRNAs against LSR and CLDN-1. The results are presented as a histogram. (C) Immunostaining for LSR and CLDN-1 in Sawano cells transfected with or without siRNAs against LSR and CLDN-1. Scale bar, $20 \mu \mathrm{m}$. LSR, lipolysis-stimulated lipoprotein receptor; CLDN, claudin; MT1, membrane-type 1; MMP, metalloproteinase; siRNA, small interfering RNA.

of LSR was inhibited, as revealed by western blotting and immunostaining (Fig. 2B and C). In addition, the upregulation of cell invasion induced by the knockdown of LSR was also inhibited, whereas the knockdown of CLDN-1 alone did not affect cell invasion (Fig. 3A).

Expression of LSR and CLDN-1 on the invasive fronts of human endometrial carcinoma tissues. The expression of LSR and CLDN-1 on the invasive front of human endometrial carcinoma was examined using hematoxylin and eosin and immunohistochemical staining on paraffin sections. A decrease of LSR protein and increase of CLDN-1 protein were observed on the invasive front of endometrial carcinoma, whereas in the gland-like structure, LSR expression was high (Fig. 3B).

\section{Discussion}

In the present study, it was demonstrated that the downregulation of LSR enhanced cell invasion via CLDN-1-mediated MMPs in human endometrial cancer. LSR expression in endometrial cancer cells was reduced; this is assumed to be 
A
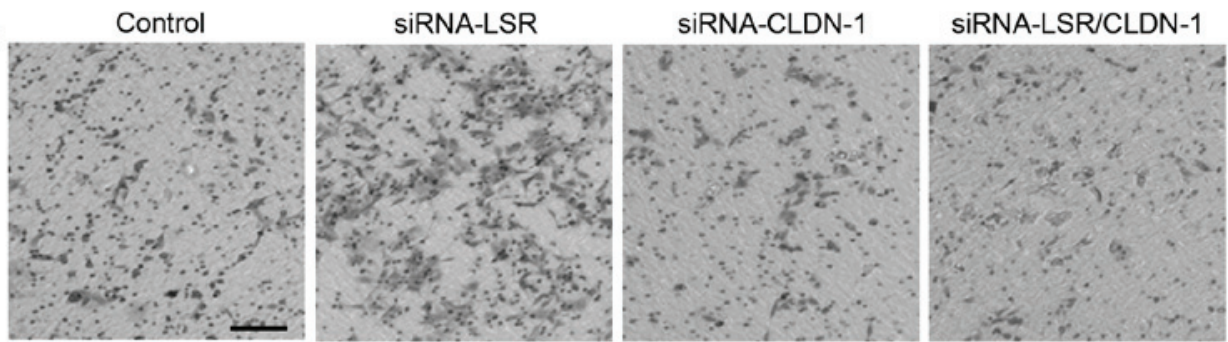

25
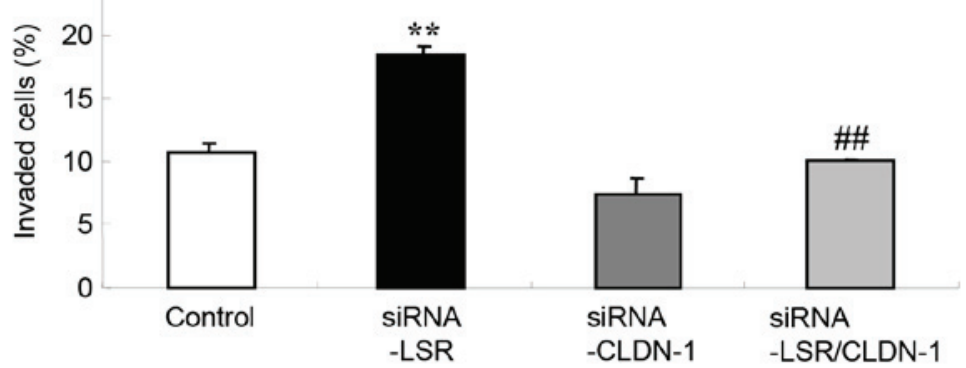

B
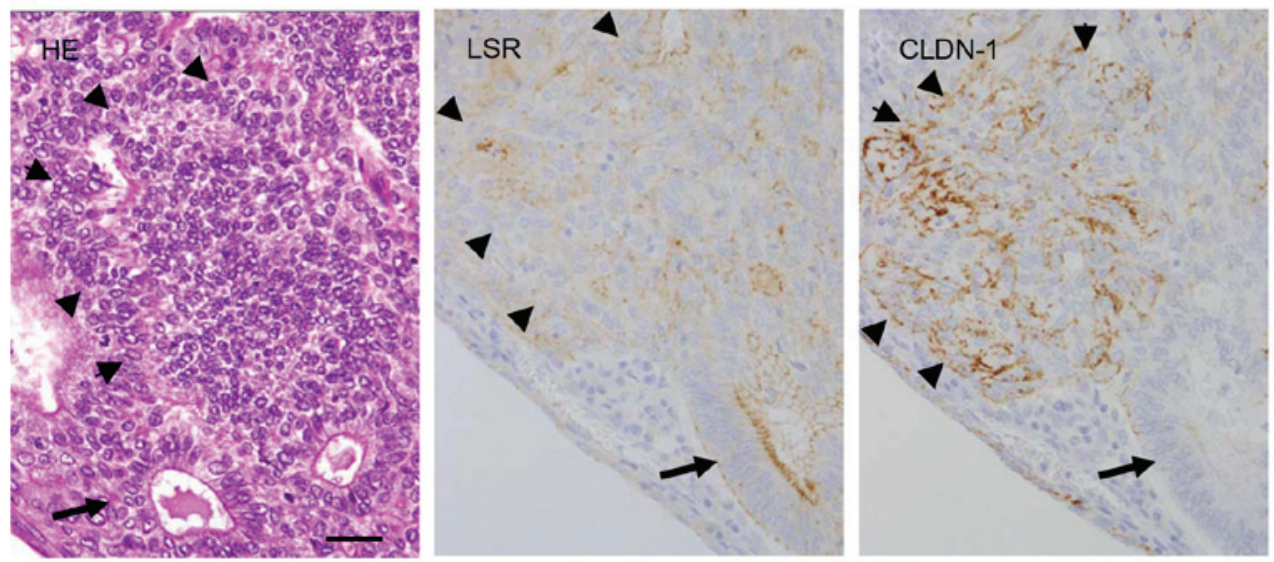

Figure 3. (A) Matrigel invasion assay of Sawano cells transfected with or without siRNAs of lipolysis-stimulated lipoprotein receptor (LSR) and claudin-1 (CLDN-1). Scale bar, $200 \mu \mathrm{m}$. The results are presented below as a histogram. Control vs. siRNA-LSR, ${ }^{* *} \mathrm{P}<0.01$; siRNA-LSR vs. siRNAs-LSR/CLDN-1, ${ }^{\# \prime} \mathrm{P}<0.01$. (B) $\mathrm{H} \& \mathrm{E}$ and immunohistochemical staining for LSR and CLDN-1 in human endometrial carcinoma (arrowheads indicate the invasive front; arrows indicate the gland-like structure). Scale bar, $100 \mu \mathrm{m}$. siRNA, small interfering RNA; LSR, lipolysis-stimulated lipoprotein receptor; CLDN, claudin; H\&E, hematoxylin and eosin.

associated with poor prognosis, as a low LSR protein level was observed in the invasive front, and a high level in the gland-like structures (Fig. 3B). However, little is yet known about the detailed alterations of LSR expression during the carcinogenesis of endometrial carcinoma. A low level of the bTJ protein CLDN-7 is associated with a late tumor stage and low histological grade in endometrial carcinoma (7). In intrahepatic cholangiocarcinoma, expression of the tTJ protein TRIC decreases in parallel with dedifferentiation (19). TRIC expression is associated with Snail-induced EMT in gastric carcinoma cells and shows a negative correlation with the degree of differentiation in pancreatic ductal adenocarcinomas $(13,14)$. The expression of the tTJ protein LSR may be associated with tumor progression and the degree of differentiation in addition to TRIC (16).

In the present study, the knockdown of LSR by the siRNA in Sawano cells was observed to induce cell invasion. Loss of the bTJ proteins OCLN and CLDNs in certain types of cancer enhances cell invasion $(6,20)$. Downregulation of
CLDN-7 promotes cell invasion in endometrial cancer (7). It is reported that knockdown of LSR increases cell motility and invasion in bladder cancer cells (15). In the present study, the detailed mechanisms of how loss of LSR promoted cell invasion in endometrial cancer were investigated. Knockdown of LSR induced CLDN-1 expression and Sp1 activity in the CLDN-1 promoter region in Sawano cells. Furthermore, in LSR-knockdown Sawano cells, the mRNA levels of MMP-1, MMP-2 and MMP-10, and the protein levels of MT1-MMP, MMP-2, MMP-9 and MMP-10 were increased. Knockdown of CLDN-1 by siRNA prevented the upregulation of cell invasion induced by knockdown of LSR in the cells. CLDNs are regulated via various transcriptional factors (21) and the CLDN-1 promoter region contains an Sp1 binding site (18). In oral squamous cell carcinoma, CLDN-1 upregulates cancer cell invasion activity through activation of MT1-MMP and MMP-2, which results in enhanced cleavage of laminin-5 $\gamma^{2}$ chains (9). CLDN-1 appears to contribute to melanoma cell invasion via increased MMP-2 secretion and activation (10). 
These findings suggest that loss of LSR signaling enhances CLDN-1 expression via Sp1 activity and upregulation of CLDN-1 subsequently promotes cell invasion through activation of MMPs. In the present study, it remained unclear how loss of LSR enhanced the Sp1 activity. Recently, the involvement of the Hippo/YAP pathway in the development and progression of endometrial cancer was reported (22). It is possible that the loss of LSR may induce cell invasion via the Hippo/YAP pathway as well as angiomotin/merlin in endometrial cancer (23).

However, in the present study, on the invasive front of human endometrial carcinoma in vivo, a decrease of LSR and increase of CLDN-1 were observed. These findings suggest that downregulation of LSR may induce malignancy, including by inducing cell invasion via upregulation of CLDN-1 in human endometrial carcinoma in vivo.

Taken together, the results of this study suggested a novel mechanism in which downregulation of the tTJ protein LSR promotes cell invasion in endometrial cancer. The mechanism is important for studying the association of tTJs with the cellular invasion of cancer.

\section{Acknowledgements}

This study was supported by the Ministry of Education, Culture, Sports, Science, and Technology, and the Ministry of Health, Labour and Welfare of Japan.

\section{References}

1. Ikenouchi J, Furuse M, Furuse K, Sasaki H, Tsukita S and Tsukita S: Tricellulin constitutes a novel barrier at tricellular contacts of epithelial cells. J Cell Biol 171: 939-945, 2005.

2. Masuda S, Oda Y, Sasaki H, Ikenouchi J, Higashi T, Akashi M, Nishi E and Furuse M: LSR defines cell corners for tricellular tight junction formation in epithelial cells. J Cell Sci 124: 548-555, 2011.

3. Matter K and Balda MS: Signaling to and from tight junctions. Nat Rev Mol Cell Biol 4: 225-236, 2003.

4. Tsukita S, Yamazaki Y, Katsuno T, Tamura A and Tsukita S: Tight junction-based epithelial microenvironment and cell proliferation. Oncogene 27: 6930-6938, 2008.

5. Martin TA and Jiang WG: Loss of tight junction barrier function and its role in cancer metastasis. Biochim Biophys Acta 1788: 872-891, 2009.

6. Martin TA: The role of tight junctions in cancer metastasis. Semin Cell Dev Biol 36: 224-231, 2014.

7. Li X, Li Y, Qiu H and Wang Y: Downregulation of claudin-7 potentiates cellular proliferation and invasion in endometrial cancer. Oncol Lett 6: 101-105, 2013.

8. Leech AO, Cruz RG, Hill AD and Hopkins AM: Paradigms lost-an emerging role for over-expression of tight junction adhesion proteins in cancer pathogenesis. Ann Transl Med 3: 184, 2015 .
9. Oku N, Sasabe E, Ueta E, Yamamoto T and Osaki T: Tight junction protein claudin-1 enhances the invasive activity of oral squamous cell carcinoma cells by promoting cleavage of laminin-5 gamma2 chain via matrix metalloproteinase (MMP)-2 and membrane-type MMP-1. Cancer Res 66: 5251-5257, 2006

10. Leotlela PD, Wade MS, Duray PH, Rhode MJ, Brown HF, Rosenthal DT, Dissanayake SK, Earley R, Indig FE, Nickoloff BJ, et al: Claudin-1 overexpression in melanoma is regulated by PKC and contributes to melanoma cell motility. Oncogene 26: 3846-3856, 2007.

11. Kondoh A, Takano K, Kojima T, Ohkuni T, Kamekura R, Ogasawara N, Go M, Sawada N and Himi T: Altered expression of claudin-1, claudin-7 and tricellulin regardless of human papilloma virus infection in human tonsillar squamous cell carcinoma. Acta Otolaryngol 131: 861-868, 2011.

12. Patonai A, Erdélyi-Belle B, Korompay A, Somorácz A, Straub BK, Schirmacher P, Kovalszky I, Lotz G, Kiss A and Schaff Z: Claudins and tricellulin in fibrolamellar hepatocellular carcinoma. Virchows Arch 458: 679-688, 2011.

13. Korompay A, Borka K, Lotz G, Somorácz A, Törzsök P, Erdélyi-Belle B, Kenessey I, Baranyai Z, Zsoldos F, Kupcsulik P, et al: Tricellulin expression in normal and neoplastic human pancreas. Histopathology 60: E76-E86, 2012.

14. Masuda R, Semba S, Mizuuchi E, Yanagihara K and Yokozaki H: Negative regulation of the tight junction protein tricellulin by snail-induced epithelial-mesenchymal transition in gastric carcinoma cells. Pathobiology 77: 106-113, 2010.

15. Herbsleb M, Birkenkamp-Demtroder K, Thykjaer T, Wiuf C, Hein AM, Orntoft TF and Dyrskjøt L: Increased cell motility and invasion upon knockdown of lipolysis stimulated lipoprotein receptor (LSR) in SW780 bladder cancer cells. BMC Med Genomics 1: 31, 2008

16. Reaves DK, Fagan-Solis KD, Dunphy K, Oliver SD, Scott DW and Fleming JM: The role of lipolysis stimulated lipoprotein receptor in breast cancer and directing breast cancer cell behavior. PLoS One 9: e91747, 2014.

17. Kurman RJ, Carcangiu ML, Herrington CS and Young RH: WHO Classification of tumours of Female Reproductive Organs. 4th edition. Lyon, France, International Agency for Research on Cancer, 2014.

18. Wang HB, Wang PY, Wang X, Wan YL and Liu YC: Butyrate enhances intestinal epithelial barrier function via up-regulation of tight junction protein Claudin-1 transcription. Dig Dis Sci 57: 3126-3135, 2012 .

19. Somorácz A, Korompay A, Törzsök P, Patonai A, Erdélyi-Belle B, Lotz G, Schaff Z and Kiss A: Tricellulin expression and its prognostic significance in primary liver carcinomas. Pathol Oncol Res 20: 755-764, 2014

20. Süren D, Yıldırım M, Kaya V, Alikanoğlu AS, Bülbüller N, Yildiz M and Sezer C: Loss of tight junction proteins (Claudin 1,4 and 7) correlates with aggressive behavior in colorectal carcinoma. Med Sci Monit 20: 1255-1262, 2014.

21. Khan $\mathrm{N}$ and Asif AR: Transcriptional regulators of claudins in epithelial tight junctions. Mediators Inflamm 2015: 219843, 2015.

22. Romero-Pérez L, Garcia-Sanz P, Mota A, Leskelä S, Hergueta-Redondo M, Díaz-Martín J, López-García MA, Castilla MA, Martínez-Ramírez A, Soslow RA, et al: A role for the transducer of the Hippo pathway, TAZ, in the development of aggressive types of endometrial cancer. Mod Pathol 28: 1492-1503, 2015.

23. Moleirinho S, Guerrant W and Kissil JL: The Angiomotins-from discovery to function. FEBS Lett 588: 2693-2703, 2014. 